\section{P205 BURDEN OF ANXIETY AND DEPRESSION IN THE DIFFICULT ASTHMA CLINIC AND RELATIONSHIP TO OUTCOME}

'JSS Cannie, ${ }^{2}$ Bryce, ${ }^{2} \mathrm{G}$ MacDonald, 'D Cowan, 'M Brewis. 'Glasgow Royal Infirmary, Glasgow, UK; ${ }^{2}$ Stobhill Ambulatory Care Hospital, Glasgow, UK

10.1136/thoraxjnl-2017-210983.347

National Review of Asthma Deaths (NRAD) ${ }^{1}$ stated that "there is a well-recognised link between asthma and psychosocial problems; the prevalences of anxiety, depression and panic disorder are much higher in people with asthma than in matched controls and are associated with poor outcomes." We aim to evaluate the prevalence of psychological illness within our difficult asthma clinic and its impact on asthma outcomes using data from the BTS Difficult Asthma Registry.

Methods Retrospective analysis of data submitted to the BTS Difficult Asthma Registry from July 2009 to June 2016. Outcomes were compared in those with and without anxiety or depression as defined by Hospital Anxiety and Depression (HAD) scores for each of $\geq 11 / 21$. Categories for comparison included Asthma Control Questionnaire (ACQ) and Asthma Quality of Life Questionnaire (AQLQ) scores, Forced expiratory volume in one second (FEV1), Fraction of exhaled nitric oxide (FeNO), Unplanned GP/A+E attendances, Hospital Admissions, and need for oral prednisolone. Simple statistical tests such as Mann-Whitney and unpaired $t$ tests were used to analyse the data.

Results The database included 198 individuals (68 male and 130 female), of whom 69 (35\%) had anxiety and $49(25 \%)$ had depression. There were no statistically significant differences between each group for FEV1, FeNO and peripheral eosinophil count. Anxiety was associated with higher ACQ and lower AQLQ scores than non-anxiety (4 vs 2.8 and 2.9 vs 3.8 respectively, both $\mathrm{p}<0.0001)$ and increased number of steroid boosts per year (6 vs $5, \mathrm{p}=0.013$ ). Depression was associated with higher ACQ and lower AQLQ scores than nondepression $(p<0.0001)$ and increased number of steroid boosts per year ( 6 vs $5, \mathrm{p}=0.021)$.

Conclusions There is a high prevalence of anxiety and depression in the difficult asthma clinic, and both co-morbidities are associated with a greater symptom burden and an increased number of steroid boosts per year not explained by objective measures of asthma control. Further research is required to evaluate the impact of clinical psychology in this setting.

\section{REFERENCE}

1. NRAD - https://www.rcplondon.ac.uk/projects/outputs/why-asthma-still-kills

\section{P206 A STUDY TO INVESTIGATE THE MECHANISMS UNDERLYING CIRCADIAN RHYTHM IN ASTHMA}

HJ Durrington, K Krakowiak, D Singh, D Ray. University of Manchester, Manchester, UK

\subsection{6/thoraxjnl-2017-210983.348}

Background Time of day is critical in the pathogenesis of asthma, and has been realised for centuries. Symptoms of asthma are worse around $4 \mathrm{am}$, when airway restriction is at its highest. Many asthma treatments are taken in the morning or evening, however there is increasing data that steroids are more efficacious if taken mid-afternoon. Investigating the biological timing of asthma is crucial to better understand the pathogenesis of asthma, this may lead to the discovery of new drug targets, and the identification of dynamic biomarkers that change by time of day and would be useful in a future chronotherapeutics study.

Aims - Define new biochemical pathways involved in the circadian variation in asthma

- Determine a circadian biomarker in asthma

Method We recruited 10 atopic, moderately severe asthmatics and 10 healthy volunteers to complete 4 study visits, including an overnight stay. Blood, induced sputum and breath were sampled at intervals throughout the day and night, and physiological measurements made. PBMCs were harvested from peripheral blood at 4 am and $4 \mathrm{pm}$ and plated in 6 groups, control,+LPS/anti-CD3/anti CD28,+P38 i,+LPS/anti-CD3/anti CD28+P381,+Dexamethasone, +LPS/anti-CD3/anti CD28 +dexamethasone for 2 hours. Conditioned medium was collected and frozen at -80 , cell lysates were prepared for RNA extraction, and protein purification.

REC reference 14/NW/1352.

Results There is a high amplitude circadian change in $\mathrm{FEV}_{1}$ in asthmatics compared to healthy controls. The nadir is at 4 am (figure 1). There was also an increase in sputum and serum eosinophils (a key effector cell in asthma) at 4 am compared to $4 \mathrm{pm}$ in asthmatics (figure1).

On-going workflow includes

- Transcript measurement from PBMCs using a combination of RNA-Sequencing and nanostring technology (for clock genes; inflammatory mediators; Signalling regulators; MAP kinase family members)

- Bioplex screen for expression and activation of additional MAPkinase components, and IkBa, from protein extracts and conditioned media

- Lipidomic analyses of serial, matched serum samples will allow analysis of the ceramide/sphingolipid pathway

- Breathomics analysis for volatile organic compounds in serial, matched breath samples.

Discussion We have demonstrated a significant diurnal effect on asthma lung physiology and eosinophil profiles. Further downstream analysis of serum, breath and sputum is underway.

\section{P207 AMBIENT AIR POLLUTION AND ADMISSIONS TO HOSPITAL WITH EXACERBATIONS OF ASTHMA}

F Thompson, A Hujan, M Richardson, G Woltmann, S Siddiqui, S Gonem. University of Leicester, Leicester, UK

10.1136/thoraxjnl-2017-210983.349

Background Air pollution has been linked to increased morbidity and mortality associated with a number of chronic health conditions including asthma. We hypothesised that levels of ambient air pollution would be related to the number of hospital admissions with exacerbations of asthma.

Methods Data on asthma admissions to a large acute NHS trust in the East Midlands were extracted using discharge diagnosis codes over a five-year period from April 2011 to March 2016. Ambient air pollution levels during this period were obtained from the website of the Department for Environment, Food and Rural Affairs. These data were based on a single monitoring station located in the centre of the City which recorded hourly readings of ozone, nitrogen oxides and 